B.G. Basmaji

Nagoya Math. J.

Vol. 35 (1969), 99-107

\title{
MONOMIAL REPRESENTATIONS AND METABELIAN GROUPS ${ }^{1)}$
}

\author{
B.G. BASMAJI
}

1. Introduction. In this paper we develop a method to find all the irreducible and inequivalent representations over the complex field $C$ of a family of finite groups that includes the metabelian groups. The outline of the paper is as follows: In $\S 2$ we let $P$ be a one-dimensional representation of a subgroup $H$ of a finite group $G$ and find a maximal subgroup $K$, $H \subseteq K \subseteq G$, such that an extension $\bar{P}$ of $P$ to $K$ exists. We show that the induced representation $\bar{P}^{G}$ is irreducible if $K$ is normal in $G$. In $\S 3$ we give all the irreducible and inequivalent representations of the "generalized metabelian group" $G$, and in particular of the metabelian group, and decompose the group ring $C G$ into its simple components. The convenience of this method is shown in $\S 4$ where we determine the representations of the metacyclic group and a metabelian group of order $2 s p^{2}, p$ an odd prime and $s \mid p-1$. The algorithm in $\$ 5$ is supplementary and can be used to find representations of more general groups than the two in $\$ 4$.

In this paper we make use of theorems 45.2 and 45.6 of Curtis-Reiner [3, §45], in the special case (due to Shoda [6]) where $L, L_{1}, L_{2}$ afford onedimensional representations.

2. Induced representations. Let $G$ be a finite group and $H, D$, and $K$ be subgroups of $G$ such that $G \supseteq K \supseteq H \supseteq D$ with the following conditions:

(i) $D$ is normal in $H$ and $H / D$ is cyclic.

(ii) $K^{\prime} \cap H \subseteq D$ where $K^{\prime}$ is the commutator group of $K$.

Received June 12, 1968

1) This paper is essentially Chapter III of the author's dissertation submitted to the University of Maryland for the Ph.D. degree. The author wishes to thank Professor Kuroda for his advice. Also, this research was partially supported by NSF grants GP-1837 and GP-6010. 
(iii) $K$ is maximal in the sense that if $K_{1} \supseteq K$ and $K_{1}^{\prime} \cap H \subseteq D$, then $K_{1}=K$.

(iv) $K$ is normal in $G$.

Let $H$ and $D$ be fixed. Then $K$ depends on $H$ and $D$. Sometimes, where confusion is likely to occur, we let $K=K(H, D)$. Note that we can always find a $K$ with conditions (ii) and (iii), but not necessarily with conditions (ii), (iii), and (iv).

Now let $A=\left\{H, K^{\prime}\right\}$ and $B=\left\{D, K^{\prime}\right\}$. Then if $H=D+a D+\cdots+a^{s-1} D$, we may set $A=B+a B+\cdots+a^{s-1} B$. Since $K / A$ is abelian we let $z_{1} A$, $z_{2} A, \cdots, z_{n} A$ be a basis for $K / A$. Then for each $z_{i}$ there exists a smallest positive integer $t_{i}$ such that $z_{i}^{t_{i}} \in A$.

Let $\zeta$ be a primitive $s$-th root of unity and $P$ be a one-dimensional representation of $A$ with kernel $B$ such that $P(a)=\zeta$. There exist $\varphi(s)=$ $\varphi([H: 1])$ distinct such representations of $A$ with kernel $B$. Let $\theta_{i}, i=1$, $\cdots, n$, be a solution of

$$
\theta^{t_{i}}=P\left(z_{i}^{t_{i}}\right) .
$$

Each $k \in K$ is uniquely expressed as $k=z_{1}^{e_{1}} \cdots z_{n}^{e_{n}} h, h \in A, 0 \leqslant e_{i} \leqslant t_{i}-1$. Set

$$
\bar{P}(k)=\theta_{1}^{e_{1}} \cdots \theta_{n}^{e_{n}} P(h) .
$$

Then $\bar{P}$ is a one-dimensional representation of $K$ and is afforded by the minimal left ideal $(C K) \bar{e}$ where $\bar{e}$ is given by

$$
\bar{e}=[K: 1]^{-1} \sum_{k \in K} \bar{P}\left(k^{-1}\right) k .
$$

For each $P$ there exist $[K: A]$ distinct such extensions $\bar{P}$.

For $x \in G$, the left ideal $(C K) \bar{e}_{x}$, where

$$
\bar{e}_{x}=[K: 1]^{-1} \sum_{k \in K} \bar{P}\left(x^{-1} k^{-1} x\right) k,
$$

affords the representation $\bar{P}_{x}$ where $\bar{P}_{x}(k)=\bar{P}\left(x^{-1} k x\right)$ for all $k \in K$. We call $\bar{P}$ and $\bar{P}_{x} G$-conjugate representations and $\overline{\boldsymbol{e}}$ and $\overline{\boldsymbol{e}}_{x} \quad G$-conjugate idémpotents.

Let $R(H, D, K)$ be the set of all representations $\bar{P}$ for every possible $P$ with kernel $D$ and their $G$-conjugates and $I(H, D, K)$ be the set of all idempotents $\bar{e}$ and their $G$-conjugates. Note that $K^{\prime} \cap x^{-1} H x \subseteq x^{-1} D x$ for all 
$x \in G$ and is maximal in the sense that $K \supseteq x^{-1} H x$ and $K^{\prime} \cap x^{-1} H x \subseteq x^{-1} D x$. Hence $R\left(x^{-1} H x, x^{-1} D x, K\right)=R(H, D, K)$ and $I\left(x^{-1} H x, x^{-1} D x, K\right)=I(H, D, K)$.

Lemma. (a) If $\bar{P} \in R(H, D, K)$, then $\bar{P}^{G}$ is an irreducible representation of $G$ afforded by the minimal left ideal $(C G) \bar{e}$ of $C G$. Moreover $(C G) \bar{e}$ is a direct summand of the simple component $(C G) \tilde{e}$ of $C G$ where $\tilde{e}$ is the sum of the distinct $G$-conjugates of $\bar{e}$.

(b) If $\bar{P}$ and $\bar{P}^{\prime}$ are in $R(H, D, K)$, then $\bar{P}^{G}$ and $\bar{P}^{\prime G}$ are equivalent if and only if $\bar{P}$ and $\bar{P}^{\prime}$ are $G$-conjugate.

(c) Assume that $H$ is normal in $G, D$ is not conjugate to $D_{1}(\subseteq H), \bar{P} \in R(H, D, K)$ and $\bar{P}_{1} \in R\left(H, D_{1}, K_{1}\right)$, where $K_{1}$ is similarly defined. Then $\bar{P}^{G}$ and $\bar{P}_{1}^{G}$ are inequivalent.

Proof. (a) Let $x \notin K$ and assume that $\bar{P}(k)=\bar{P}\left(x^{-1} k x\right)$ for all $k \in K$. Then $k^{-1} x^{-1} k x$ is in the kernel $\bar{D}$ of $\bar{P}$. Let $K_{1}=\{K, x\}$, then $K_{1}^{\prime} \subseteq \bar{D}$. But $\bar{D} \cap H=D$ or $K_{1} \supsetneq K \supseteq H$ and $K_{1}^{\prime} \cap H \subseteq D$, a contradiction since $K$ is maximal. Hence $\bar{P}(k) \neq \bar{P}\left(x^{-1} k x\right)$ for at least one $k \in K$. From [3, (45.2)], $\bar{P}^{G}$ is irreducible. Now $\bar{P}^{G}$ is of degree $[G: K]$. Let $\bar{\chi}^{G}$ be the character of $\bar{P}^{G}$ then $\bar{\chi}^{G}(g)=0$ for all $g \notin K$ and $\bar{\chi}^{G}(k)=\sum \bar{P}_{x}(k)$ where $x$ runs over a complete set of coset representatives of $K$ by $G$. The central idempotent $\tilde{e}$ is given by

$$
\tilde{e}=\frac{[G: K]}{[G: 1]} \sum_{g \equiv G} \overline{\bar{\chi}^{G}(g)} g
$$

Hence it can be easily shown that $\tilde{e}=\sum_{x} \bar{e}_{x}$ where $x$ runs over a complete set of coset representatives of $G$ by $K$. For $x \notin K$, we have $\sum_{k \in K} P\left(k^{-1}\right) P\left(x^{-1} k x\right)$ $=0$, and hence $\bar{e} \cdot \bar{e}_{x}=0$. Hence $(C G) \tilde{e}$ is the direct sum of the $[G: K]$ left ideals $(C G) \bar{e}_{x}$ or each $(C G) \bar{e}_{x}$ is a minimal left ideal and $(C G) \bar{e}$ affords $\bar{P}^{a}$.

Parts (b) and (c) follow using (a) above and [3, (45.6)] which completes the proof.

Let $R_{c}(H, D, K)$ and $I_{c}(H, D, K)$ be complete sets of representatives of the conjugate classes of $R(H, D, K)$ and $I(H, D, K)$ respectively. From the above lemma any two elements of $R_{c}(H, D, K)$ induce inequivalent representations of $G$ and any element of $R(H, D, K)$ induces a representation equivalent to some representation induced by an element of $R_{c}(H, D, K)$. Let $H$ be normal in $G$ and $\nu(D)=\left[G: N_{G}(D)\right]$. Then the number of elements of $R_{c}(H, D, K)$ is 
$\nu(D) \varphi([H: D])[K: A] /[G: K]$.

In case $K^{\prime} \subseteq D$ we have $A=H$.

3. The generalized metabelian groups. We first give a

Definition. A finite group $G$ is called a generalized metabelian group if $G$ has a normal (invariant) series $E=A_{0} \subsetneq A_{1} \mp \cdots \subsetneq A_{n+1}=G$, where $E=\{1\}$, satisfying the following conditions:

(i) $A_{i} / A_{i-1}$ is abelian for $i=1,2, \cdots, n+1$.

(ii) For any subgroup $D_{i}$ of $G$ such that $A_{i-1} \subseteq D_{i} \subsetneq A_{i}$ when $1 \leqslant i \leqslant n-1$ and $A_{n-1} \subseteq D_{n} \subseteq A_{n}$, with $A_{i} / D_{i}$ cyclic, there exists a subgroup $K_{i}=K\left(A_{i}, D_{i}\right) \supseteq A_{i}$ of $G$ such that $K_{i}^{\prime} \subseteq D_{i}$ and $K_{i}$ satisfies (iii) and (iv) of $\S 2$.

Using the derived series, $E=G^{\prime \prime} \subseteq G^{\prime} \subseteq G$, of a finite metabelian group $G$, we can easily show that $G$ is a generalized metabelian group. We give an example of a group $G$ which is not metabelian but is generalized metabelian. In Blackburn [2], the existence of a $p$-group ( $p$ odd) $G=\{x, y, z\}$ whose derived group $G=\{a, b\}$ is metacyclic is proved. The defining relations of a special case is as follows:

$$
z^{p^{3}}=y^{p^{3}}=x^{p^{2}}=a^{p^{2}}=b^{p^{3}}=1
$$

with $x^{-1} y^{-1} x y=[x, y]=a,[y, z]=b,[a, z]=a^{p},[b, z]=b^{2 p},[a, y]=b^{p},[b, x]=$ $a^{-p} b^{p 2},[a, b]=b^{p 2}$, and $[b, y]=[a, x]=[z, x]=1$. The derived series of $G$ is $\{1\} \subseteq\left\{b^{p 2}\right\} \subseteq\{a, b\} \subseteq G$. Condition (i) in the definition is trivially satisfied. Let $K=\left\{a, b^{p}, x, y^{p 2}, z^{p}\right\}$. Then $K$ is normal in $G, K^{\prime}=\{1\}, K^{\prime} \cap G^{\prime \prime}=\{1\}$, and $K$ is maximal in the sense that $K \supseteq G^{\prime \prime}$ and $K^{\prime} \cap G^{\prime \prime} \subseteq D=\{1\}$. Hence (ii) of the definition above is satisfied for $\{1\} \subseteq D \subsetneq\left\{b^{p 2}\right\}$. The proof for the remaining cases follows since $G /\left\{b^{p 2}\right\}$ is metabelian. Hence $G$ is a generalized metabelian group.

Let $D_{1}, \cdots, D_{t}$ form the set of all subgroups of a finite abelian group $A$ such that $A / D_{i}$ is cyclic. Then, from the self-duality of $A$, all the cyclic subgroups of $A$ are $C_{1}, \cdots, C_{t}$ with $C_{i} \cong A / D_{i}, i=1, \cdots, t$. If $T_{i}$ is the set of generators of $C_{i}$, then the set theoritic union $\bigcup_{i=1}^{t} T_{i}=A$ and therefore

$$
\sum_{i=1}^{t} \varphi\left(\left[C_{i}: 1\right]\right)=\sum_{i=1}^{t} \varphi\left(\left[A: D_{i}\right]\right)=[A: 1]
$$


Using the above result and the notations of the definition we prove,

Theorem. Let $G$ be a generalized metabelian group with the normal series given as in the above definition. Then all the irreducible inequivalent representations of $G$ are given by the set of all $\bar{P}^{G}$ with $\bar{P} \in \bigcup_{i=1}^{n}\left\{\cup^{(i)} R_{c}\left(A_{i}, D_{i}, K_{i}\right)\right\}$ and the union $\cup^{(i)}$ is over all $D_{i}$ which are non-conjugate in $G$.

Proof. Applying the Lemma $\bar{P}^{G}$ is irreducible and any $\bar{P}^{G}$ and $\bar{P}^{\prime G}$, with $\bar{P} \neq \bar{P}^{\prime}$, are inequivalent. The number of elements of $R_{c}\left(A_{i}, D_{i}, K_{i}\right)$ is

$$
\nu\left(D_{i}\right) \varphi\left(\left[A_{i}: D_{i}\right]\right)\left[K_{i}: A_{i}\right] /\left[G: K_{i}\right] .
$$

Each $\bar{P}^{G}$ for $\bar{P} \in R_{c}\left(A_{i}, D_{i}, K_{i}\right)$ is of degree $\left[G: K_{i}\right]$. Hence multiplying the above number by $\left[G: K_{i}\right]^{2}$ and summing over all possible $D_{i}$ 's given in the definition we have for $i=1,2, \cdots, n-1$

$$
\begin{aligned}
\Sigma^{\prime} \nu & \left(D_{i}\right) \varphi\left(\left[A_{i}: D_{i}\right]\right)\left[G: A_{i}\right] \\
& =\left[\sum \varphi\left(\left[A_{i}: D_{i}\right]\right)\right]\left[G: A_{i}\right] \\
& =\left(\left[A_{i}: A_{i-1}\right]-1\right)\left[G: A_{i}\right]=\left[G: A_{i-1}\right]-\left[G: A_{i}\right],
\end{aligned}
$$

where the first summation $\Sigma^{\prime}$ is over all non-conjugate $D_{i}$ 's such that $A_{i-1} \subseteq D_{i} \subseteq A_{i}, A_{i} / D_{i}$ cyclic, and the second summation $\Sigma$ is over all $D_{i}$ 's such that $A_{i-1} \subseteq D_{i} \subsetneq A_{i}$ and $A_{i} / D_{i}$ cyclic. For $i=n$ the sum is [G: $A_{n}$ ]. Hence adding all the above quantities for $1 \leqslant i \leqslant n$ we get $[G: 1]$, the order of $G$. This completes the proof.

Corollary. Let $G$ be as in the above theorem. Then $C G=\Sigma(C G) \bar{e}$ is a decomposition of $C G$ as a direct sum of its minimal left ideals where $\bar{e}$ runs over all elements of the set $\bigcup_{i=1}^{n}\left\{\cup^{(i)} I\left(A_{i}, D_{i}, K_{i}\right)\right\}$ where the union $\cup^{(i)}$ is over all $D_{i}$, for fixed $i$, which are non-conjugate in $G$.

Using the lemma, we may set $\tilde{e}$ as the sum of distinct conjugates of $\bar{e}$, and hence we have $C G=\Sigma(C G) \tilde{e}$, a decomposition of $C G$ into its simple components.

Corollary. Let $G$ be a finite metabelian group, $A$ a normal abelian subgroup, and $G / A$ abelian. Then all the irreducible inequivalent representations of $G$ are given by the set of all $\bar{P}^{G}$ where $P \in \cup R_{c}(A, D, K)$ and the union is taken over all non-conjugate subgroups $D$ of $A$ such that $A / D$ is cyclic.

The above result for the case where $G$ splits over $A$ was proved by Berman [1]. 
COROLlary. Every irreducible representation of a generalized metabelian group $G$ is equivalent to a monomial representation.

Another proof for the above corollary for the case where $G$ is metabelian is given in [3, §52]. Still another proof can be given by applying a result on faithful representations of metabelian groups proved by Shoda [6], which was also differently proved by Itô [4]. Below we generalize this result of Shoda to the generalized metabelian group.

Corollary. Let $G$ be a generalized metabelian group with the normal series as in the definition and assume $G$ has an irreducible faithful representation. Then all the irreducible faithful representations $S$ of $G$ have the same degree $d$, and all the maximal abelian subgroups $K$, containing $A_{1}$, which are normal in $G$ have the same index $t$, and $t=d$. Moreover $S$ is equivalent to an induced representation $\bar{P}^{G}$ where $\bar{P}$ is some one-dimensional representation of any $K$.

Proof. From the theorem, the faithfulness of $S$ implies that there exists a subgroup $D_{1}, A_{1} / D_{1}$ is cyclic and $\bigcap_{x \in G} x^{-1} D_{1} x=\{1\}$. Hence $K=K\left(A_{1}, D_{1}\right)$ is abelian and the result follows.

4. Applications. The methods of the previous two sections are used to find all the representations of the groups below. The symbols $P, \bar{P}, D$, $K, \bar{e}$, and $\tilde{e}$ have the same meanings as before and sometimes a subscript is added whenever confusion is likely to occur. To avoid repetition the calculations are omitted.

(i) Representations of the metacyclic group. This is also done by Munkholm [5].

Let $G$ be a metacyclic group with the defining relations

$$
a^{n}=b^{m}=1, a^{k}=b^{t}, b^{-1} a b=a^{r}
$$

where $t \mid m$ and $k r-k \equiv r^{t}-1 \equiv 0(\bmod n)$. Consider the normal series $\{1\} \subseteq\{a\}$ $\subseteq\{a, b\}=G$ and let $D_{s}=\left\{a^{s}\right\}, s \mid n$. Then $K_{s}=K\left(\{a\}, D_{s}\right)=\left\{a, b^{t_{s}}\right\}$ where $t_{s}$ is the smallest positive integer such that $r^{t_{s}} \equiv 1(\bmod s)$. Let $M_{s}$ be the multiplicative group of the reduced residues modulo $s$ and consider $R_{s}=\{r\}$ as a subgroup of $M_{s}$. Let $\zeta_{s}$ be a primitive $s$-th root of unity, $P_{s}(a)=\zeta_{s}^{y}$, $(y, s)=1$, and $\theta_{s}^{(i)}, i=1, \cdots, t / t_{s}$, set of all solutions of $\theta^{t / t_{s}}=\zeta_{s}^{y k}$. - Also, let $\bar{P}_{s}(a)=P_{s}(a)$ and $\bar{P}_{s}\left(b^{t_{s}}\right)=\theta_{s}^{(i)}$. Then all the irreducible inequivalent representations of $G$ are $\bar{P}_{s}^{G}$ where $s$ runs over all divisors of $n, y$ runs over 
a complete set of coset representatives of $M_{s}$ by $R_{s}$ and $i=1, \cdots, t / t_{s}$. Also, we have

$$
\bar{e}_{s}=\bar{e}_{s}^{(y)}=t /\left(n t_{s}\right)_{\alpha=0}^{n-1} \sum_{\beta=0}^{t / t_{s}-1} \zeta_{s}^{-y \alpha}\left(\theta_{s}^{(i)}\right)^{-\beta} a^{\alpha} b^{t s \beta} \text {, and } \tilde{e}_{s}=\sum_{j=0}^{t_{s}-1} \bar{e}_{s}^{\left(y r^{\prime}\right)}
$$

(ii) Representations of a group of order $2 s p^{2}$. Let $p$ be an odd prime, $(r, p)=1, \quad r \neq 1(\bmod p)$, and $s$ the smallest positive integer such that $r^{s} \equiv 1(\bmod p)$. Let $G=\{a, b, x, y\}$ be the group with the following defining relations:

$$
\begin{aligned}
& a^{p}=b^{p}=x^{s}=y^{2}=a^{-1} b^{-1} a b=x^{-1} y^{-1} x y=1 \\
& x^{-1} a x=a^{r}, x^{-1} b x=b^{r}, y^{-1} a y=b, \text { and } y^{-1} b y=a .
\end{aligned}
$$

Then $G^{\prime}=\{a, b\}$ and $G^{\prime \prime}=\{1\}$. Let $\zeta$ be a primitive $p$-th root of unity. We list below a complete set of non-conjugate subgroups $D, G^{\prime} / D$ cyclic, and for each $D$ we find the corresponding $\bar{P}, \bar{e}$, and $\tilde{e}$.

(1) $D=\{b\}, D=\left\{b a^{g^{i}}\right\}, i=1,2, \cdots,(p-1) / 2-1$ where $g$ is a primitive root $\bmod p$. Here $K=K\left(G^{\prime}, D\right)=G^{\prime}$ and $P(a)=\zeta^{t},(t, p)=1$, and $P(d)=1$ for $d \in D$. The $(p-1) / s$ irreducible inequivalent representations $P^{G}$ of degree $2 s$ are given by letting $t$ run over a complete set of coset representatives of $M_{p}=\{g\}$ by $R_{p}=\{r\}$. The idempotents corresponding to $D_{1}=\{b\}$ and $D_{2}=\left\{b a^{g^{i}}\right\}$ are

$$
\bar{e}_{1}=\bar{e}_{1}^{(t)}(a, b)=p^{-2} \sum_{\alpha=0}^{p-1} \sum_{\beta=0}^{p-1} \zeta^{-t \alpha} a^{\alpha} b^{\beta} \text { and } \bar{e}_{2}=\bar{e}_{2}^{(t)}(a, b)=p^{-2} \sum_{\alpha=0}^{p-1} \sum_{\beta=0}^{p-1} \zeta^{t\left(g^{i} \alpha-\beta\right)} a^{\alpha} b^{\beta}
$$

respectively and $\tilde{e}_{j}=\sum_{k=0}^{s-1}\left[\bar{e}_{j}^{\left(t r^{k}\right)}(a, b)+\bar{e}_{j}^{\left(t r^{k}\right)}(b, a)\right], j=1,2$.

(2) $D=\{a b\}$ where $s$ is odd. Here $K=G^{\prime}, P(a)=\zeta^{t}$ and $P(a b)=1$ where $(t, p)=1$. The $(p-1) / 2 s$ irreducible inequivalent representations $P^{G}$ of degree $2 s$ are given by letting $t$ run over a complete set of coset representatives of $M_{p}$ by $R_{1}=\{-r\}$. Here

$$
\bar{e}=\bar{e}^{(t)}=p^{-2} \sum_{\alpha=0}^{p-1} \sum_{\beta=0}^{p-1} \zeta^{t(\alpha-\beta)} a^{x} b^{\beta} \text { and } \tilde{e}=\sum_{k=0}^{s-1}\left[\bar{e}^{\left(t r^{k}\right)}+\bar{e}^{\left(-t r^{k}\right)}\right] .
$$

(3) $D=\{a b\}$ where $s$ is even. Here $K=\left\{G^{\prime}, y x^{s / 2}\right\}, P(a)=\zeta^{t}$ and $P(a b)=1$ where $(t, p)=1$. We have $\bar{P}_{i}(h)=P(h)$ for $h \in G^{\prime}$ and $\bar{P}_{i}\left(y x^{s / 2}\right)=$ $(-1)^{i}, i=1,2$. The $(p-1) / s$ irreducible inequivalent representations $\bar{P}^{G}$, 
$i=1,2$, of degree $s$ are given by letting $t$ run over a complete set of coset representatives of $M_{p}=\{g\}$ by $R_{p}=\{r\}$. Also

$$
\bar{e}_{i}=\bar{e}_{i}^{(t)}=1 /\left(2 p^{2}\right) \sum_{\alpha=0}^{p-1} \sum_{\beta=0}^{p-1} \zeta^{t(\alpha-\beta)} a^{\alpha} b^{\beta}\left[1+(-1)^{i} y x^{s / 2}\right] \text { and } \tilde{e}_{i}=\sum_{k=0}^{s-1} \bar{e}_{i}{ }^{\left(t r^{k}\right)}
$$

(4) $D=\left\{a b^{-1}\right\}$. Exactly as (3) with $y$ exchanged for $y x^{s / 2}, P\left(a b^{-1}\right)=1$ for $P(a b)=1$, and $\zeta^{-t(\alpha+\beta)}$ for $\zeta^{t(\beta-\alpha)}$.

(5) $D=G^{\prime} . \quad K=G$ and we get $2 s$ one-dimensional representations of G.

5. Cyclic factor groups. In this section we give an algorithm to find all the subgroups $D$ of a finite abelian group $A$, with $A / D$ cyclic. Clearly we only need to give this for the case where $A$ is an abelian $p$ group.

Algorithm. Let $A=\left\{a_{1}, \cdots, a_{n}\right\}$ be an abelian $p$-group of type $\left(\alpha_{1}, \cdots, \alpha_{n}\right), 1 \leqslant \alpha_{1} \leqslant \cdots \leqslant \alpha_{n}, a_{i}^{p^{\alpha}}=1$. Let $B=\left\{a_{1}, \cdots, a_{n-1}\right\}$ and assume $D$ is a subgroup of $B$ such that $B / D$ is cyclic of order $p^{\alpha}, 0 \leqslant \alpha \leqslant \alpha_{n-1} \leqslant \alpha_{n}$, and set

$$
B=D+a D+\cdots+a^{p^{\alpha}-1} D .
$$

If $\alpha=\alpha_{n}$ then all the distinct subgroups $\bar{D}$ of $A$ such that $A / \bar{D}$ is cyclic and $B \cap \bar{D}=D$ are given by $\bar{D}=\left\{a_{n} a^{k}, D\right\}, k=0,1, \cdots, p^{\alpha}-1$, and we may set $A=\bar{D}+a \bar{D}+\cdots+a^{p^{\alpha}-1} \bar{D}$.

If $\alpha<\alpha_{n}$ then, in addition to the subgroups $\vec{D}$ above, we have $\bar{D}=\left\{a_{n}^{p^{\sigma} k} a, D\right\}, \quad \sigma=1, \cdots, \alpha_{n}-\alpha, \quad 1 \leqslant k \leqslant p^{\alpha}, \quad(k, p)=1$, and we may set $A=\bar{D}+a_{n} \bar{D}+\cdots+a_{n}^{p^{\alpha+\sigma}-1} \bar{D}$.

Proof. Since $D=\bar{D} \cap B$, the smallest positive power of $a$ in $\bar{D}$ is $a^{p^{\alpha}}$. If $d \in \bar{D}$ then $d=a_{n}^{x} a^{y} d^{\prime}, d^{\prime} \in D$. If $p / x$, for some $x$, then it can be shown that $\bar{D}=\left\{a_{n} a^{k}, D\right\}$. If $p \mid x$ for every $x$, then $p / y$ for some $y$ and $\alpha<\alpha_{n}$. The results follow after some calculations. Note that when $D$ runs over all subgroups of $B, B / D$ cyclic, $\bar{D}$ runs over all subgroups of $A$, $A / \bar{D}$ cyclic.

\section{BIBLIOGRAPHY}

[1] Berman, S. On the representations of the semi-direct product of two abelian groups. Dokl. Akad, Nauk. 98 (1954), 177-180. 
[2] Blackburn, N. On prime power groups in which the derived group has two generators. Proc. Cambridge Philo. Soc. 23 (1957), 19-27.

[ 3 ] Curtis, C. and Reiner, I. "Representation Theory of Finite Groups and Associative Algebras." Interscience, New York, 1962.

[ 4 ] Itô, N. On monomial representations of finite groups. Osaka Math. J. 6 (1954), 119-127.

[5] Munkholm, H. Induced monomial representations, young elements, and metacyclic groups. Proc. Amer. Math. Soc. 19 (1968), 453-458.

[6] Shoda, K. Über die monomialen Darstellungen einer endlichen Gruppe. Proc. Phys. Math. Japan 15 (1933), 249-257.

University of Nebraska 